\title{
Gene Transfer or Gene Delivery Agent
}

National Cancer Institute

\section{Source}

National Cancer Institute. Gene Transfer or Gene Delivery Agent. NCI Thesaurus. Code C61199.

Genetic engineered recombinant products, including viral or non-viral based system that can be used to transfer genetic materials of interest into cells for either research or clinical purpose. 